\title{
Some Relations between Isologic and Varietal Perfect Groups
}

\author{
Shokufeh Lotfi and S. Mostafa Taheri \\ Faculty of Mathematics, Golestan University, Gorgan, Iran \\ Correspondence should be addressed to Shokufeh Lotfi; sh.lotfi@gu.ac.ir \\ Received 26 July 2016; Accepted 15 November 2016 \\ Academic Editor: Shaofang Hong
}

Copyright (C) 2016 S. Lotfi and S. M. Taheri. This is an open access article distributed under the Creative Commons Attribution License, which permits unrestricted use, distribution, and reproduction in any medium, provided the original work is properly cited.

In 1940, Hall introduced the notion of $V$-isologism, with respect to a given variety of groups 9 . In the present article, we study the concepts of $V$-perfect groups, $V$-subgroup, and $V$-quotient irreducible groups, with respect to a given variety of groups $\vartheta$. Also we prove and obtain some results.

\section{Introduction}

In 1940, Hall introduced the notion of isoclinism, which is an equivalence relation on the class of all groups such that all abelian groups fall into an equivalence class. This notion is weaker than isomorphism and plays an important role in classification of finite $p$-groups in [1]. Later he generalized the notion of isoclinism to the notion of $V$-isologism, with respect to a given variety of groups $\vartheta$ in [2]. If $\vartheta$ is the variety of all the trivial groups, abelian groups, or nilpotent groups of class at most $n$, then $V$-isologism coincides with isomorphism, isoclinism, and $n$-isoclinism, respectively; for more information see $[1,3]$. The purpose of this article is to show some properties of $V$-perfect groups, $V$-subgroup, and $V$-quotient irreducible groups, with respect to a given variety of groups $\vartheta$.

Throughout the paper, we assume that $\vartheta$ is the variety of groups defined by the set of words $V$ and the notations are taken from $[4] . V(G)$ denotes the verbal subgroup and $V^{*}(G)$ the marginal subgroup of $G$ with respect to $\vartheta$; see [5] for more information on varieties of groups.

For a group $G$ with a normal subgroup $N,\left[N V^{*} G\right]$ is defined (following [6]) to be the subgroup of $G$ generated by the following set:

$$
\begin{aligned}
& \left\{v\left(g_{1}, \ldots, g_{i} n, \ldots, g_{r}\right)\left(v\left(g_{1}, \ldots, g_{r}\right)\right)^{-1} \mid 1 \leq i \leq r ; v\right. \\
& \left.\quad \in V ; g_{1}, \ldots, g_{r} \in G ; n \in N\right\} .
\end{aligned}
$$

One may easily show that $\left[N V^{*} G\right]$ is the smallest normal subgroup $T$ of $G$ contained in $N$, such that $N / T \subseteq V^{*}(G / T)$.

The following results give basic properties of the verbal and the marginal subgroups of a group $G$ with respect to the variety $\vartheta$, which is useful in our investigations; see [7] for more information.

Proposition 1 (see [7, Proposition 2.3]). Let 9 be a variety of groups and $N$ be a normal subgroup of a group $G$. Then the following statements hold:

(i) $V\left(V^{*}(G)\right)=1, V^{*}(G / V(G))=G / V(G)$.

(ii) $V(G)=1 \Leftrightarrow V^{*}(G)=G \Leftrightarrow G \in \mathcal{V}$.

(iii) $\left[N V^{*} G\right]=1 \Leftrightarrow N \subseteq V^{*}(G)$.

(iv) $V(G / N)=V(G) N / N, V^{*}(G) N / N \subseteq V^{*}(G / N)$.

(v) $V(N) \subseteq\left[N V^{*} G\right] \subseteq N \cap V(G)$, in particular $V(G)=$ $\left[G V^{*} G\right]$.

(vi) If $N \cap V(G)=1$, then $N \subseteq V^{*}(G)$ and $V^{*}(G / N)=$ $V^{*}(G) / N$.

(vii) If $[G, N] \subseteq V^{*}(G)$, then $[V(G), N]=1$, in particular $\left[V(G), V^{*}(G)\right]=1$.

Theorem 2 (see [7, Theorem 2.4]). Let 9 be a variety of groups and $G$ be a group with a subgroup $H$ and a normal subgroup $N$. If $G=H N$, then $V(G)=V(H)\left[N V^{*} G\right]$. 

tions.

The following definition from [3] is vital in our investiga-

Definition 3. Let $\vartheta$ be a variety of groups defined by the set of laws $V$ and let $G$ and $H$ be two groups. Then $(\alpha, \beta)$ is said to be a $V$-isologism between $G$ and $H$, if

$$
\begin{gathered}
\alpha: \frac{G}{V^{*}(G)} \longrightarrow \frac{H}{V^{*}(H)}, \\
\beta: V(G) \longrightarrow V(H)
\end{gathered}
$$

are isomorphisms such that, for all $\nu\left(x_{1}, \ldots, x_{r}\right) \in V$ and all $g_{1}, \ldots, g_{r} \in G$, we have $\beta\left(\nu\left(g_{1}, \ldots, g_{r}\right)\right)=v\left(h_{1}, \ldots, h_{r}\right)$, whenever $h_{i} \in \alpha\left(g_{i} V^{*}(G)\right)$ for $i=1, \ldots, r$. In this case, we write $G \sim H$ and we will say that $G$ is $V$-isologic to $H$.

In particular, if $\vartheta$ is the variety of abelian groups we obtain the notion of isoclinism due to Hall [1].

The following lemma is needed in our investigations. For more information see Lemma 4.4 of [7].

Lemma 4. Let $\vartheta$ be a variety of groups and $H, N$ are a subgroup and normal subgroup of group $G$, respectively. Then the following statements hold:

(i) $H \sim H V^{*}(G)$. In particular, if $G=H V^{*}(G)$, then $G \sim H$. Conversely, if $G / V^{*}(G)$ satisfies the descending chain condition on subgroups and $G \sim H$, then $G=$ $H V^{*}(G)$.

(ii) $G / N \sim G /(N \cap V(G))$. In particular, if $N \cap V(G)=$ 1 , then $G \sim G / N$. Conversely, if $V(G)$ satisfies the ascending chain condition on normal subgroups and $G \sim G / N$, then $N \cap V(G)=1$.

Now, by Lemma 4, we obtain the following theorem.

Theorem 5. Let $\vartheta$ be a variety of groups and $H$ be a subgroup of a group $G$. If $\beta$ is an epimorphism from $G$ onto $H$, then $\beta$ induces $a V$-isologism between $G$ and $H$ if and only if $(\operatorname{Ker} \beta) \cap$ $V(G)=1$.

Proof. One notes that Lemma 4 (ii) gives the "if" part. Now assume that $\beta$ induces a $V$-isologism between $G$ and $H$; then $\left.\beta\right|_{V(G)}: V(G) \rightarrow V(H)$ is an isomorphism. Hence $(\operatorname{Ker} \beta) \cap$ $V(G)=1$.

\section{2. $V$-Perfect Groups}

This section is devoted to study $V$-perfect groups, which are vital in our investigations.

The following definition is essential in our further study.

Definition 6. A group $G$ is said to be $V$-perfect with respect to the variety $\vartheta$, if $G=V(G)$.

In particular, if $\vartheta$ is the variety of abelian groups, then $V$ perfect groups coincide with perfect groups.

The following theorems give the connections between $V$ perfect and $V$-isologism groups.
Theorem 7. Let $\vartheta$ be a variety of groups and $G$ be a finite $V$-perfect group with trivial marginal subgroup. Then any $V$ isologic group $H$ to $G$ is isomorphic to the direct product of $G$ by the marginal subgroup of $H$.

Proof. By the assumption,

$$
\begin{aligned}
\frac{H}{V^{*}(H)} \cong \frac{G}{V^{*}(G)} \cong G \sim H, \\
V(H) \cong V(G)=G .
\end{aligned}
$$

Now using Lemma 4, we have $H=V(H) V^{*}(H)$ and $V^{*}(H) \cap$ $V(H)=1$. Hence $H \cong G \times V^{*}(H)$.

Theorem 8. Let $\vartheta$ be a variety of groups and $G$ be a finite group. If $H$ is a $V$-perfect subgroup of $G$ such that $H \sim G$, then $G=$ $V(G) V^{*}(G)$.

Proof. By Lemma 4, we have $G=H V^{*}(G)$ and $H=V(H)$. Hence $G=V(G) V^{*}(G)$.

Theorem 9. Let $\vartheta$ be a variety of groups and $G$ be a finite $V$-perfect group. Then $G$ can not be $V$-isologic to any proper subgroup or factor group of itself.

Proof. If $H$ is a subgroup of $G$ such that $G \sim H$, then, by Lemma 4, it follows that $N \cap V(G)=1$. Hence $N=1$.

Theorem 10. Let $G$ be a finite group and $H$ be a group of the same order and isologic to $G$, with respect to a given variety $\vartheta$. If $G$ is $V$-perfect or $V^{*}(G)=1$, then $G \cong H$.

Proof. By the definition of isologism, we have the following isomorphisms:

$$
\begin{gathered}
\alpha: \frac{G}{V^{*}(G)} \longrightarrow \frac{H}{V^{*}(H)}, \\
\beta: V(G) \longrightarrow V(H) .
\end{gathered}
$$

Now, clearly if $G=V(G)$, then $|G|=|V(H)|$. Since $|G|=|H|$, it implies that $H=V(H)$ and hence $G \cong H$. If $V^{*}(G)=1$, then the result follows immediately.

\section{Product of Varieties}

In 1976, Leedham-Green and Mckay [6] introduced the notion of the product of varieties as follows.

Let $\vartheta$ and $v$ be varieties of groups defined by the set of words $V$ and $U$, respectively. The product $\omega=v * \vartheta$ is the variety of all groups $G$ such that $U(G) \subseteq V^{*}(G)$. They also showed that the verbal subgroup of the product $\omega=v * \vartheta$ is $W(G)=\left[U(G) V^{*} G\right]$.

In this section, using the notion of the product of varieties we present some results. Also further information about product of varieties and varietal isologism may be found in [8-12].

The following theorem gives a set of defining words for this product variety. It can be used in computations with the words. For more information see ([6, Proposition 1.5]). 
Theorem 11. Using the above notations and definitions, assume $u\left(x_{1}, \ldots, x_{r}\right)$ and $v\left(x_{1}, \ldots, x_{s}\right)$ are words, and for each $i(1 \leq i \leq s)$

$$
\begin{aligned}
v_{(i)} u & \\
= & v\left(x_{1}, \ldots, x_{i-1}, x_{i} u\left(x_{s+1}, \ldots, x_{s+r}\right), x_{i+1}, \ldots, x_{s}\right) \\
\cdot & \left(v\left(x_{1}, \ldots, x_{s}\right)\right)^{-1}, \\
v^{(i)} u & =v\left(x_{1}, \ldots, x_{i-1}, u\left(x_{s+1}, \ldots, x_{s+r}\right), x_{i+1}, \ldots, x_{s}\right) \\
\cdot & \left(v\left(x_{1}, \ldots, x_{i-1}, 1, x_{i+1}, \ldots, x_{s}\right)\right)^{-1} .
\end{aligned}
$$

Then $v * \vartheta$ is defined by each of the following sets:

$$
\begin{aligned}
& W \\
& \quad=\left\{v_{(i)} u \mid v\left(x_{1}, \ldots, x_{s}\right) \in V, u \in U, 1 \leq i \leq s<\infty\right\} ; \\
& W^{\prime} \\
& \quad=\left\{v^{(i)} u \mid v\left(x_{1}, \ldots, x_{s}\right) \in V, u \in U, 1 \leq i \leq s<\infty\right\} .
\end{aligned}
$$

With respect to the marginal subgroup of a group $G$ corresponding to the variety $v * \vartheta$, we have the following theorem.

Theorem 12 (see [7, Theorem 3.2]). Let $v$ and 9 be two varieties and put $\omega=v * \vartheta$. Then for any group $G$ the following statements hold:

(i) $V^{*}(G) \subseteq W^{*}(G)$.

(ii) $W^{*}(G) / V^{*}(G) \subseteq U^{*}\left(G / V^{*}(G)\right) \subseteq W^{*}\left(G / V^{*}(G)\right)$.

Leedham-Green and Mckay proved that this product * featuring in $v * \vartheta$ is not commutative in [6]. Also, Hekster proved that this product is not associative in [7].

Now, considering the products of varieties, Neumann defined that the notions of $v \vee \vartheta$ are the variety whose set of laws are in $U \cap V$, and $[v, \vartheta]$ consists of all groups whose $U$-subgroups centralize $V$-subgroups.

The following lemma gives the connection of the above product varieties, which was already proved by Hekster in [7].

Lemma 13. Let $v$ and $\vartheta$ be two varieties of groups. Then $v \vee \vartheta \subseteq$ $v * \vartheta \subseteq v \vartheta$ and $v * \vartheta \subseteq[v, \vartheta]$.

Now, we are able to obtain the following theorem.

Theorem 14. Let $v$ and $\vartheta$ be two varieties of groups and $\omega=$ $v * \vartheta$. Then a group $G$ is $W$-perfect if and only if $G$ is $U$-perfect and $V$-perfect group.

Proof. Let $G$ be a $W$-perfect group. Then by Proposition 1, we have

$$
W(G)=\left[U(G) V^{*} G\right] \subseteq U(G) \cap V(G) .
$$

Therefore, $G$ is $U$-perfect and $V$-perfect group.

Conversely, if $G=U(G)=V(G)$, then

$$
W(G)=\left[U(G) V^{*} G\right]=\left[G V^{*} G\right]=V(G)=G .
$$

The following corollary is an immediate consequence of the above theorem.

Corollary 15. Let $v$ and $\vartheta$ be two varieties of groups, $\omega=$ $v * \vartheta$, and $G$ be a $U$-perfect group. Then $G \in \mathcal{\vartheta}$ if and only if $G \in \omega$.

Now by the virtue of the above products of varieties we have the following theorem.

Theorem 16. Let $v$ and $\vartheta$ be two varieties of groups and $G$ be an arbitrary group. Then we have the following:

(i) If $\omega=v * \vartheta$ and $G$ is either $U$-perfect or $V$-perfect group, then $G$ is not $W$-perfect group.

(ii) If $\omega=[v, \vartheta]$ and $G$ is $W$-perfect group, then $G$ is $U$ perfect and $V$-perfect group.

Conversely, if $G$ is $U$-perfect and $V$-perfect group, then $W(G)=G^{\prime}$, so $G$ is not necessarily a $W$-perfect group.

(iii) If $\omega=v \vee \vartheta$ or $\omega=v \vartheta$, then $G$ is $W$-perfect group if and only if $G$ is both $U$-perfect and $V$-perfect group.

Proof. (i) and (ii) can be easily obtained by the above notations and Corollary 15.

(iii) Let $\omega=v \vee \vartheta$. Then $W(G)=U(G) \cap V(G)$. Now, assume that $G$ is $W$-perfect group. So $U(G) \cap V(G)=G$, which implies that $G$ is both $U$-perfect and $V$-perfect group. The part "only if" is trivial.

Let $\omega=v \vartheta$. Then $W(G)=U(V(G))$. Now, if $G$ is $W$ perfect group, then, by Lemma 13, we have

$$
G=W(G) \subseteq\left[V(G) U^{*} G\right] \subseteq V(G) \cap U(G) .
$$

Thus $G=V(G) \cap U(G)$, and so $G$ is both $U$-perfect and $V$ perfect group. The part "only if" is trivial.

\section{4. $V$-Subgroup and $V$-Quotient Irreducible Groups}

Hekster by the work of Stroud [13] introduced the notions of subgroup irreducible groups and quotient irreducible groups in [7].

In this final section, by using the notions of subgroup irreducible groups and quotient irreducible groups and discussion of the previous sections we give and prove our main results, which are somehow similar to those given in [7].

The following definition is introduced by Hekster in [7].

Definition 17. Let $\vartheta$ be a variety of groups. A group $G$ is called subgroup irreducible with respect to $V$-isologism if $G$ contains no proper subgroup $H$ satisfying $G=H V^{*}(G)$. A group $G$ is called quotient irreducible with respect to $V$-isologism if $G$ contains no nontrivial normal subgroup $N$ satisfying $N \cap V(G)=1$.

In this article, we present the notions of $V$-subgroup irreducible groups, that is, the subgroup irreducible groups with respect to $V$-isologism, and $V$-quotient irreducible groups, 
that is, the quotient irreducible groups with respect to $V$ isologism.

The proof of the following lemma is straightforward; see Proposition 7.2 of [7] for more information.

Lemma 18. Let $\vartheta$ be a variety and suppose $V^{*}(G) \subseteq V(G)$. Then $G$ is both $V$-subgroup and $V$-quotient irreducible group.

A simple application of Zorn's lemma shows that, given a group and a variety $\vartheta$, one can always find a $V$-quotient irreducible group. Hence this establishes the following theorem.

Theorem 19. Let $\vartheta$ be a variety of groups. If $G$ is an arbitrary group, then there exists a normal subgroup $N$ of $G$ such that $G \sim G / N$ and $G / N$ is a $V$-quotient irreducible group.

Proof. Consider $\mathscr{A}=\{N \mid N$ is a normal subgroup of $G$ with $N \cap V(G)=1\}$. The set $\mathscr{A}$ is nonvoid because it contains the trivial subgroup. We define a partial ordering on $\mathscr{A}$ by inclusion and evidently, by Zorn's Lemma, we can find a maximal normal subgroup $N$ in $\mathscr{A}$. Since $N \cap V(G)=1$, it follows that $G \sim G / N$ by Lemma 4 . Now, suppose that $M / N$ is a normal subgroup of $G / N$ such that $(M / N) \cap V(G / N)=1$. Therefore, using the Dedekind's modular law and Proposition 1, we have $M \cap V(G) \subseteq N$. Since $N \cap V(G)=1$, we conclude that $M \in \mathscr{A}$. On the other hand, we have $N \subseteq M$ and so, by the maximality of $N$, it follows that $M=N$. Therefore $M / N$ is trivial and hence $G / N$ is $V$-quotient irreducible group.

Remark 20. Let $\omega=v * \vartheta$ be the product of varieties $v$ and $\vartheta$. If $G$ is a $W$-subgroup and $W$-quotient irreducible group, then one notes that $G$ is $U$-subgroup and $U$-quotient irreducible group and also $V$-subgroup and $V$-quotient irreducible group.

The following theorem gives a connection between perfect groups and subgroup and quotient irreducible groups.

Theorem 21. Let $\vartheta$ be a variety of groups. If $G$ is a $V$-perfect group, then $G$ is both $V$-subgroup and $V$-quotient irreducible group.

Proof. Suppose $G$ is a $V$-perfect group with a subgroup $H$ such that $G=H V^{*}(G)$. Therefore we conclude that $V(G)=$ $V(H)$ and hence $G=H$. It is easily verified that $G$ is $V$ quotient irreducible group.

In the following theorem we show that the property of being subgroup and quotient irreducible group is closed with respect to isologism.

Theorem 22. Let 9 be a variety of groups and $G_{1}$ and $G_{2}$ be two $V$-isologic groups. If $G_{1}$ is $V$-subgroup and $V$-quotient irreducible group, then so is $G_{2}$.

Proof. Let $N$ be a normal subgroup of $G_{1}$ such that $N \cap$ $V\left(G_{1}\right)=1$. Then we have $G_{2} \cong G_{1} / N$ by Theorem 5.1 of [7]. Hence $N \subseteq V^{*}(G)$ and $V^{*}\left(G_{1} / N\right)=V^{*}\left(G_{1}\right) / N$. Now, assume that

$$
G_{2} \cong \frac{G_{1}}{N}=\left(\frac{H}{N}\right) V^{*}\left(\frac{G_{1}}{N}\right)=\left(\frac{H}{N}\right)\left(\frac{V^{*}\left(G_{1}\right)}{N}\right) .
$$

So $G_{1}=H V^{*}\left(G_{1}\right)$, which implies that $G_{1}=H$ and hence $G_{2} \cong G_{1} / N=H / N$. One can easily check that the result holds when $G_{1}$ is assumed to be $V$-quotient irreducible group.

\section{Competing Interests}

The authors declare that they have no competing interests.

\section{References}

[1] P. Hall, “The classification of prime-power groups," Journal für die Reine und Angewandte Mathematik, vol. 152, pp. 130-141, 1940.

[2] P. Hall, "Verbal and marginal subgroups," Journal für die Reine und Angewandte Mathematik, vol. 182, pp. 130-141, 1940.

[3] N. S. Hekster, "On the structure of $n$-isoclinism classes of groups," Journal of Pure and Applied Algebra, vol. 40, no. 1, pp. 63-85, 1986.

[4] M. R. R. Moghaddam, A. R. Salemkar, and S. M. Taheri, "Baerinvariants with respect to two varieties of groups," Algebra Colloquium, vol. 8, no. 2, pp. 145-151, 2001.

[5] H. Neumann, Varieties of Groups, Springer, Berlin, Germany, 1967.

[6] C. R. Leedham-Green and S. McKay, "Baer-invariants, isologism, varietal laws and homology," Acta Mathematica, vol. 137, no. 1-2, pp. 99-150, 1976.

[7] N. S. Hekster, "Varieties of groups and isologisms," Journal of the Australian Mathematical Society, vol. 46, no. 1, pp. 22-60, 1989.

[8] M. R. R. Moghaddam and A. R. Salemkar, "Varietal isologism and covering groups," Archiv der Mathematik, vol. 75, no. 1, pp. $8-15,2000$.

[9] M. R. R. Moghaddam and A. R. Salemkar, "Schur-pair property and the structure of varietal covering groups," Bulletin of the Iranian Mathematical Society, vol. 27, no. 2, pp. 1-16, 2001.

[10] M. R. Moghaddam and A. R. Salemkar, "Relative covering groups," Communications in Algebra, vol. 31, no. 12, pp. 57695780, 2003.

[11] M. Parvizi and S. Kayvanfar, "A product of varieties in relation to Baer invariants," Indagationes Mathematicae, vol. 17, no. 2, pp. 297-309, 2006.

[12] A. R. Salemkar and F. Mirzaei, "Characterizing $n$-isoclinism classes of lie algebras," Communications in Algebra, vol. 38, no. 9, pp. 3392-3403, 2010.

[13] P. W. Stroud, "On a property of verbal and marginal subgroups," Proceedings of the Cambridge Philosophical Society, vol. 61, pp. 41-48, 1965. 


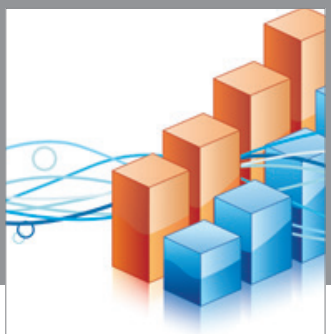

Advances in

Operations Research

vatem alat4

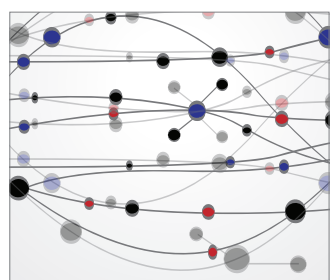

\section{The Scientific} World Journal
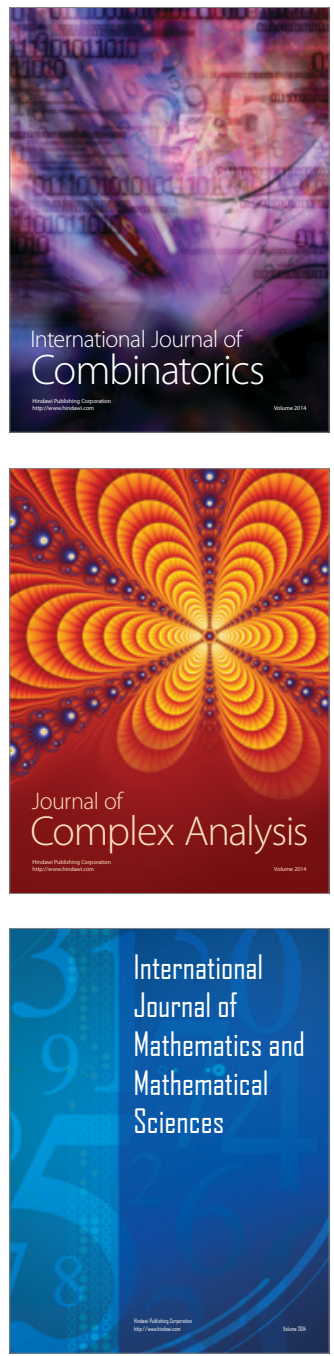
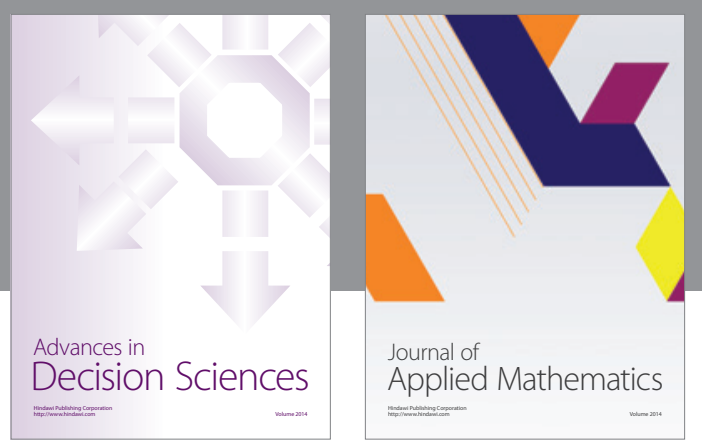

Algebra

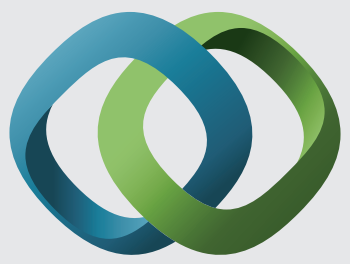

\section{Hindawi}

Submit your manuscripts at

http://www.hindawi.com
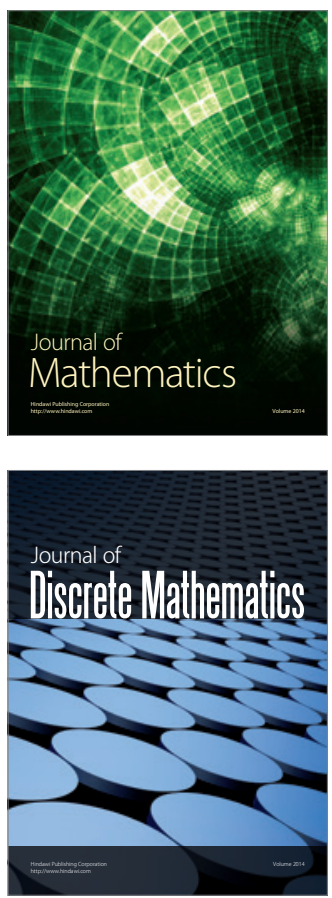

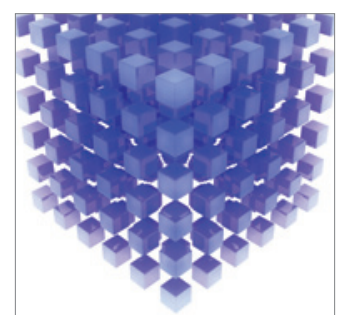

Mathematical Problems in Engineering
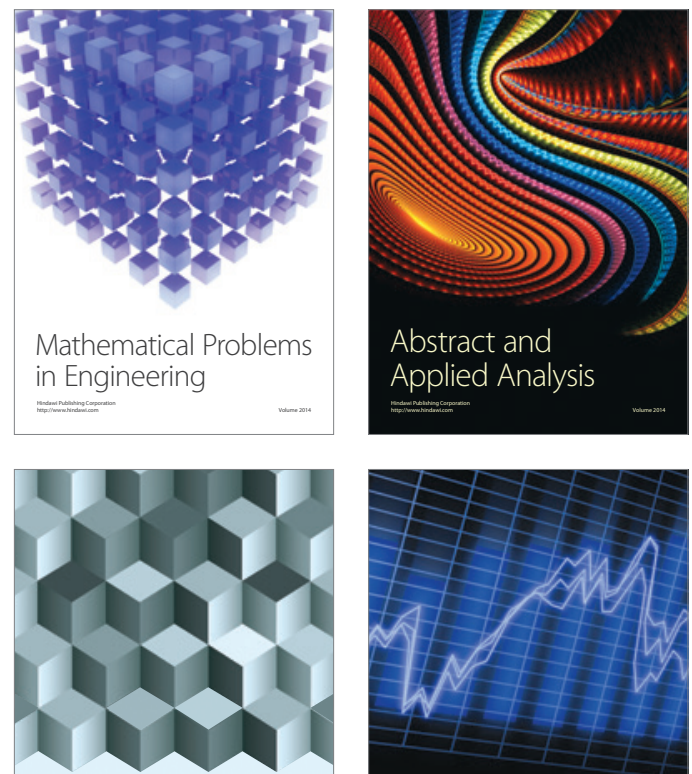

Journal of

Function Spaces

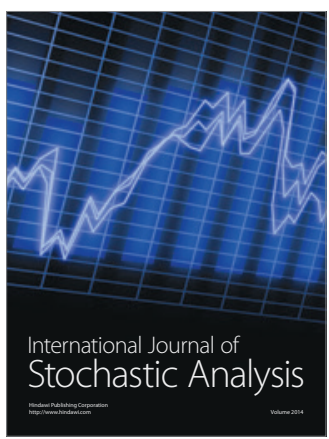

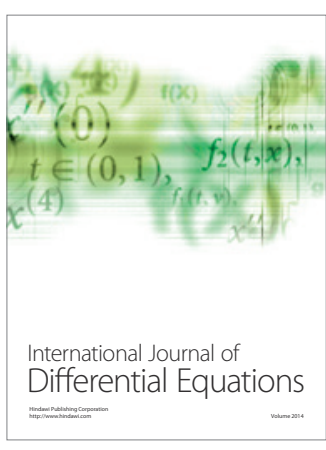
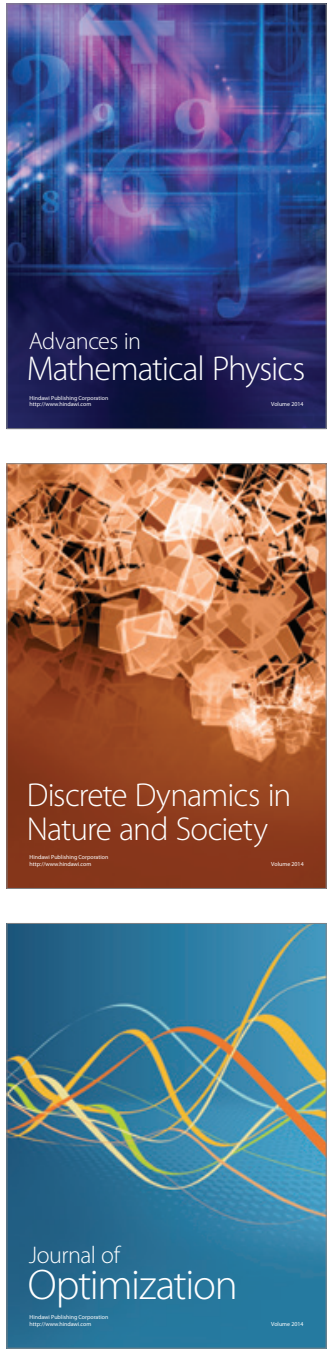